\title{
Surgical treatment of incarcerated calculi via laparoscopic bile duct exploration using laparotomy biliary lithotomy forceps
}

\author{
H. JIANG, S.Y. WANG, X.L. JIN, J.C. JIN, H.B. GU and F.M.ZHANG \\ Department of Hepatopancreatobiliary Surgery, Affiliated Hospital of Yanbian University, \\ Yanji, Jilin 133000, P.R. China
}

Received June 11, 2015; Accepted June 14, 2016

DOI: $10.3892 /$ etm.2016.3618

\begin{abstract}
The present study aimed to investigate the practicability and clinical value of applying laparotomy biliary lithotomy forceps to laparoscopic bile duct exploration (LCBDE) for the surgical treatment of incarcerated calculi. A total of 63 patients were diagnosed with cholecystolithiasis and choledocholithiasis. The present study performed a retrospective analysis of clinical samples from 16 of these patients who had incarcerated calculi at the terminus of the common bile duct, and who had been treated with laparoscopic cholecystectomy and LCBDE. During the procedure, laparotomy biliary lithotomy forceps were used to gently remove the calculi from the common bile duct. Of the surgical procedures that used laparotomy biliary lithotomy forceps, one case was unsuccessful and 15 cases were successful. The results of the present study suggested that it may be clinically advisable to use laparotomy biliary lithotomy forceps to remove incarcerated calculi from the common bile duct during a laparoscopy, since it is easy, economical and effective.
\end{abstract}

\section{Introduction}

Since the technological development of the laparoscope, laparoscopic common bile duct exploration (LCBDE) has been adopted at an increasing number of hospitals, in particular due to its advantages, including small scarring, painlessness, a quick-recovery time, short hospital stays and few complications (1). The primary aim of LCBDE is the removal of incarcerated calculi from the lower part of the common bile duct.

The present study aimed to investigate the practicability and clinical value of applying laparotomy biliary lithotomy forceps to the LCBDE protocol.

Correspondence to: Professor Xinglin Jin, Department of Hepatopancreatobiliary Surgery, Affiliated Hospital of Yanbian University, 1327 Juzi Street, Yanji, Jilin 133000, P.R. China E-mail: xljinyj@163.com

Key words: incarcerated calculi, laparoscopic bile duct exploration, laparotomy biliary lithotomy forceps

\section{Patients and methods}

Clinical data. Between August 2013 and December 2014, 63 patients, including 29 males and 34 females (male: female $=1: 1.2$ ), with choledocholithiasis were admitted to the Department of Hepatopancreatobiliary Surgery at the Affiliated Hospital of Yanbian University (Yanji, China). The patients were aged between 31 and 87 years, with an average age of 59 years. All 63 cases had been diagnosed with both cholecystolithiasis and choledocholithiasis, of which 16 cases had incarcerated calculi at the lower end of the common bile duct.

Surgical methods. General anesthesia was administered by tracheal intubation and the patient was placed in the left lateral tilt position with the head elevated. The pore for the laparoscope was established within the lower side of the navel, whereas the mail operational pore was created $2 \mathrm{~cm}$ below the xiphoid process and two auxiliary operational pores were established to the right of the midclavicular line and $2 \mathrm{~cm}$ below the costal margin of the anterior axillary line on the right side. Following localization of the common bile duct under a laparoscope, a 1-1.5 cm longitudinal incision was made close to the upper side of the duodenum. Subsequently, as a choledochoscope was used to confirm the size and position of the incarcerated calculi, another incision was made on the abdominal wall directly above the longitudinal incision of the common bile duct. Through this small incision, laparotomy biliary lithotomy forceps (Fig. 1) of the correct size and radian were inserted (Fig. 2), during which the intra-abdominal pressure was lowered to $9 \mathrm{mmHg}$ in order to reduce the distance between the abdominal wall and incision on the common bile duct. According to the anatomical structure of the common bile duct, the calculi were gently removed (Fig. 3). A choledochoscopic examination was performed to confirm that there were no residual calculi within the common bile duct. A lithotomy forcep was inserted into the incision on the abdominal wall and remained in the wall until the calculi were removed to prevent leakage. In the event of any contamination of the facilities, the forceps were washed and then removed outside of the abdominal cavity.

\section{Results}

Outcomes. The laparotomy biliary lithotomy forceps were used in 16 cases, of which only one failed. During the surgery, 


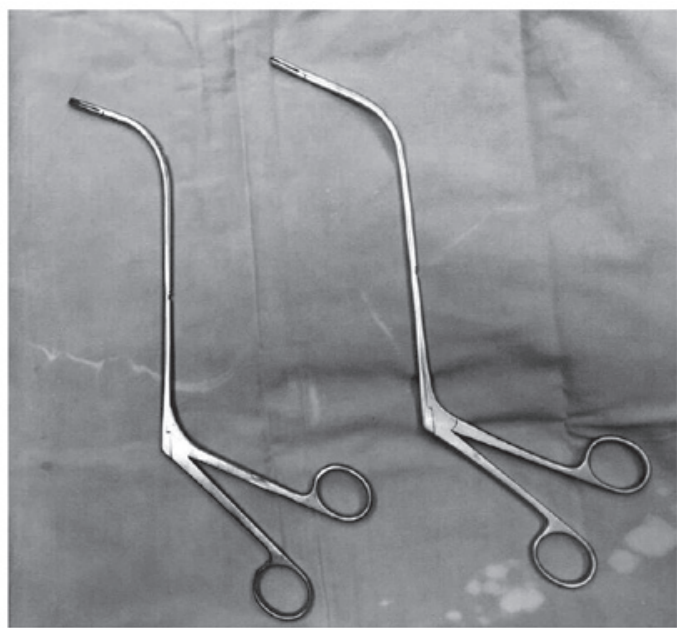

Figure 1. Laparotomy biliary lithotomy forceps.

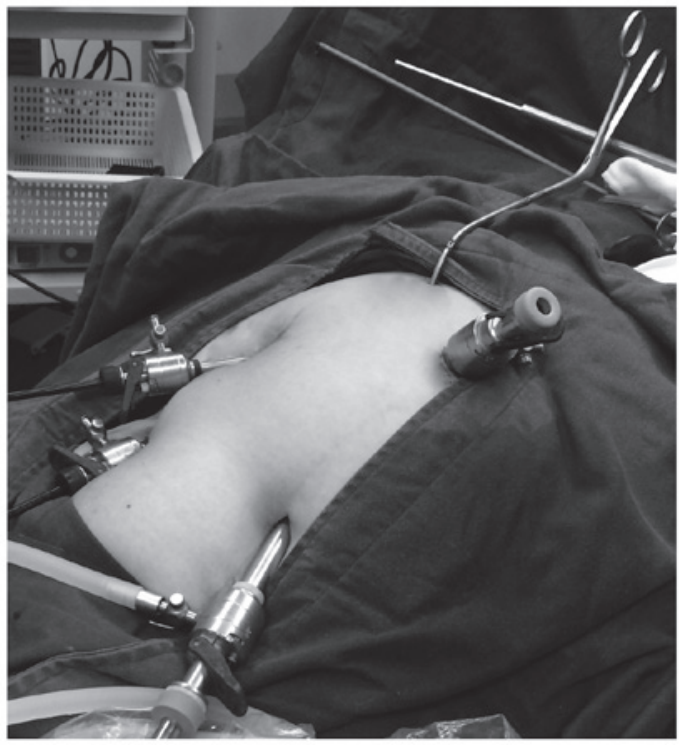

Figure 2. Laparotomy biliary lithotomy forceps of the right size and radian were inserted into a patient.

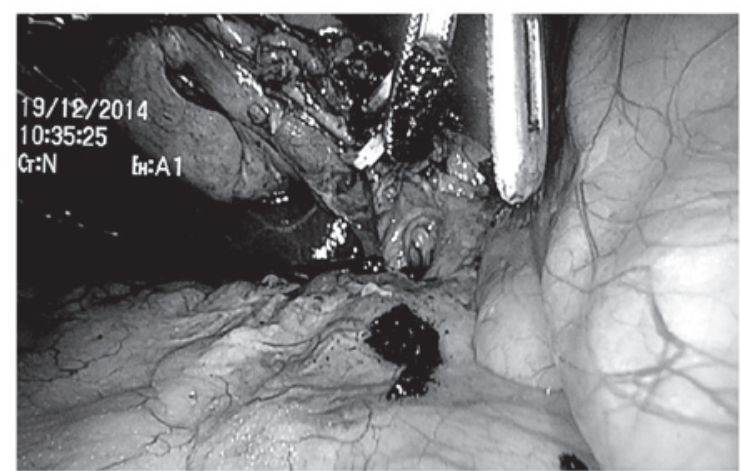

Figure 3. According to the anatomical structure of the common bile duct, the calculi were removed gently.

a T-shaped tube was inserted during the initial surgery, and after 6 weeks, the calculi were removed under a choledochoscope. The insertion of a T-shaped tube or primary suturing of the common bile duct was performed in the 15 successful cases, according to the extent of dilatation of the common bile duct and the extent of obstruction of the distal bile duct. The mean surgical duration of the 15 cases who were treated successfully was $1.5 \mathrm{~h}$, and the mean hospitalization duration post-surgery was 6 days.

\section{Discussion}

The common bile duct is divided into four segments from the top to the bottom, as follows: Duodenal upper segment, duodenal posterior segment, pancreas segment and medial wall of the duodenum. The first two segments have smooth, thin muscular walls, a wide lumen and are easily extended. The latter two sections, in particular the fourth segment, are surrounded by numerous organs and contain the sphincter of the common bile duct. Their lumens are narrow, their walls are thick and they are located in a deep and inaccessible position for dissection, therefore they are not easily extended. As compared with the third segment, the fourth segment rapidly narrows and crosses the wall of the duodenum, thus forming an obtuse angle pointing outward and down (2). This leads to the center of the lumen becoming eccentric and a diverticulum formation in the third section allows the calculi to become embedded. The wrong treatment or a surgical error during the removal of a common bile duct stone may lead to complications, including iatrogenic damage of the choledocho-pancreatico-duodenal junction and bleeding in the biliary tract and duodenum. The general treatment principles for embedded common bile duct stones are as follows: To remove the lesions, to remove all calculi, to correct the narrowness, to maintain unobstructed drainage, to prevent relapse, to reduce injury and complications, and to lower risks. Therefore, it is important to select an effective method for the treatment of patients with incarcerated calculi of the common bile duct.

It is often challenging to remove calculi from patients due to them being embedded, hard or large. The distal part of lithotomy forceps is L-shaped with two 2-cm flat indented forcep bites at the terminus. The maximum open angle of the lithotomy forceps is $70^{\circ}$, which permits contact with the nearest surface of the calculi only. However, basket extraction is also challenging, as it may be unable to pass through the lumen and subsequently unfold insufficiently to cover the calculi or it may be unable to remove a large or embedded calculi. Furthermore, the force applied during basket extraction may lead to bleeding, and basket extraction is also time-consuming. If the calculi have been incarcerated for a long time, thus becoming hard and completely obstructive, mucosal tissue on the bile duct wall may undergo hyperplasia and thickening, and this cannot be removed by lithotomy forceps. In this case, laser or machinery methods could be adopted to shatter the stone, after which the pieces may be removed (3). Basket impaction or rupture of the basket traction wire are complications unique to mechanical lithotripsy $(4,5)$, some patients cannot tolerate the prolonged lithotripsy operating time, thus, the procedure has to be conducted in several sessions $(6,7)$. Endoscopic electrohydraulic lithotripsy requires costly and fragile endoscopes (8) as well as the excellent coordination of two very experienced endoscopists. Endoscopic laser lithotripsy is the most recent method, but its use is still limited, also owing to the need for costly 
equipment (9). Recent laser lithotriptors combine the advantages of dye and solid-state lasers at a reasonably low price (10), thus potentially allowing for a future progressive diffusion of laser lithotripsy worldwide $(10,11)$. The critical determining factors for the success of extracorporeal shock-wave lithotripsy (ESWL) are stone size/structure (12) and CBD diameter (13). ESWL is at present not considered as being the first line treatment for difficult bile duct stones (9).

The length and radian of the laparotomy biliary lithotomy forceps are more suitable for the structure of the common bile duct than that of the laparoscopic biliary lithotomy forceps. Experienced surgeons are able to perform the surgery as gently and carefully as a general laparotomy, such that iatrogenic biliary tract damage may be rare. The essential protocols performed during these type of surgery are summarized, as follows: i) The incision should be made directly above the bile duct incision on the abdominal wall to reduce the distance to the distal part of the biliary tract and to prevent damage to the biliary tract; ii) pneumoperitoneum pressure should be reduced to $9 \mathrm{mmHg}$ in order to shorten the distance between the abdominal wall and bile duct incision; and iii) lithotomy forceps should be inserted through the incision in the abdominal wall, left in the wall after the calculi are removed to prevent leakage and removed when the surgery is completed. If there is contamination of the facilities, they should be washed and then be removed outside of the abdominal cavity. Furthermore, the process should be performed under the supervision of a laparoscope, and lithotomy should be guided according to pre-operative images and choledochoscope examinations performed intraoperatively, thus ensuring that the surgical process runs smoothly. In addition, when the incarcerated calculi are dense, forceps should be used initially to smash the calculi, followed by removal. The surgeons that perform the operation should be experienced in laparotomy since they should not make contact with any organs during the LCBDE.

In conclusion, the present study demonstrated that laparotomy biliary lithotomy forceps may be effectively used in LCBDE for the surgical treatment of incarcerated calculi, in particular in basic-level hospitals. The numerous advantages of this include surgical ease, short operation and hospitalization time, and low costs.

\section{References}

1. Noble H, Tranter S, Chesworth T, Norton S and Thompson M: A randomized, clinical trial to compare endoscopic sphincterotomy and subsequent laparoscopic cholecystectomy with primary laparoscopic bile duct exploration during cholecystectomy in higher risk patients with choledocholithiasis. J Laparoendosc Tech A 19: 713-720, 2009.

2. Kune GA: Surgical anatomy of common bile duct. Arch Surg 89: 995-1004, 1964.

3. KurokiT, TajimaY, Tsuneoka N, Kitasato A, Adachi T, Kosaka T, Okamoto T, Ohno S and Kanematsu T: Placement of a plasticbiliary biliary stent tube with primary closure of the common bile duct after laparoscopic common bile duct exploration. Hepatogastroenterology 57: 1034-1036, 2010.

4. Lux G, Ell C, Hochberger J, Müller D and Demling L: The first successful endoscopic retrograde laser lithotripsy of common bile duct stones in man using a pulsed neodymium-YAG laser. Endoscopy 18: 144-145, 1986.

5. Moon JH, Cha SW, Ryu CB, Kim YS, Hong SJ, Cheon YK, Cho YD, Kim YS, Lee JS, Lee MS, et al: Endoscopic treatment of retained bile-duct stones by using a balloon catheter for electrohydraulic lithotripsy without cholangioscopy. Gastrointest Endosc 60: 562-566, 2004.

6. Cipolletta L, Costamagna G, Bianco MA, Rotondano G, Piscopo R, Mutignani M and Marmo R: Endoscopic mechanical lithotripsy of difficult common bile duct stones. Br J Surg 84: 1407-1409, 1997.

7. Garg PK, Tandon RK, Ahuja V, Makharia GK and Batra Y: Predictors of unsuccessful mechanical lithotripsy and endoscopic clearance of large bile duct stones. Gastrointest Endosc 59: 601-605, 2004.

8. Chang WH, Chu CH, Wang TE, Chen MJ and Lin CC: Outcome of simple use of mechanical lithotripsy of difficult common bile duct stones. World J Gastroenterol 11: 593-596, 2005.

9. Shim CS: How Should Biliary Stones be Managed? Gut Liver 4: 161-172, 2010

10. Hochberger J, Tex S, Maiss J and Hahn EG: Management of difficult common bile duct stones. Gastrointest Endosc Clin N Am 13: 623-634, 2003.

11. Patel SN, Rosenkranz L, Hooks B, Tarnasky PR, Raijman I, Fishman DS, Sauer BG and Kahaleh M: Holmium-yttrium aluminum garnet laser lithotripsy in the treatment of biliary calculi using single-operator cholangioscopy: A multicenter experience (with video). Gastrointest Endosc 79: 344-348, 2014.

12. Tandan M, Reddy DN, Santosh D, Reddy V, Koppuju V, Lakhtakia S, Gupta R, Ramchandani M and Rao GV: Extracorporeal shock wave lithotripsy of large difficult common bile duct stones: Efficacy and analysis of factors that favor stone fragmentation. J Gastroenterol Hepatol 24: 1370-1374, 2014.

13. Amplatz S, Piazzi L, Felder M, Comberlato M, Benvenuti S, Zancanella L, Di Fede F, de'Guelmi A, Bertozzo A, Farris P, et al. Extracorporeal shock wave lithotripsy for clearance of refractory bile duct stones. Dig Liver Dis 39: 267-272, 2007. 